\title{
SCREENING OF THE ANTIVIRAL ACTIVITY IN THE RANGE OF C5 AND N3 SUBSTITUTED 4-THIAZOLIDINONE DERIVATIVES
}

\author{
D.V.Kaminskyy \\ Danylo Halytsky Lviv National Medical University \\ Department of Pharmaceutical, Organic and Bioorganic Chemistry \\ 69, Pekarska str., Lviv-10, 79010, Ukraine.E-mail: dankaminskyy@gmail.com; dankam@meduniv. lviv.ua
}

Key words: 4-thiazolidinones; screening; antiviral activity

\begin{abstract}
Prospects for the search of antiviral agents among 4-thiazolidinone derivatives, as well as the optimal directions of the main core structure optimization - namely C5 and N3, have been described. As the result of the screening performed (within the Antimicrobial Acquisition and Coordinating Facility programme), the values of the antiviral activity of the target 5-substituted-4-thiazolidinones with the carboxylic group (or its derivatives) in the N3 residue in relation to a wide range of the viral panels have been determined. The active compounds, which can be regarded as promising structures in the anti-flu agent design, have been identified, as well as 3-\{5-[2-chloro-3-(4nitrophenyl)-allylidene]-4-oxo-2-thioxothiazolidine-3-yl\}-propionic (1) and-succinic acids (3) have been identified as hit-compounds with a marked anti-VZV activity (SI values - 27 and 38, respectively).
\end{abstract}

\begin{abstract}
СКРИНІНГ ПРОТИВІРУСНОї АКТИВНОСТІ В РЯДУ С5 ТА N3 ЗАМІЩЕНИХ ПОХІДНИХ 4-ТІАЗОЛІДИНОНІВ Д.В.Камінський

Ключові слова: 4-тіазолідинони; скриніне; противірусна активність

Показано перспективність пошуку противірусних агентів серед похідних 4-тіазолідинонів, зазначені оптимальні напрямки оптимізації структури базового гетероциклу - положення C5 та N3. У результаmі проведеного скринінгу (в рамках міжнародної програми Antimicrobial Acquisition \& Coordinating Facility) встановлено значення противірусної активності досліджуваних 5-заміщених-4-тіазолідинонів, що містять карбоксильну групу (або її похідні) в заміснику положення N3, відносно широкого спектра вірусів. Виділено ряд активних сполук, що можуть розглядатися як перспективні структури при дизайні агентів, що діють на віруси грипу, а також ідентифріковано 3-\{5-[2-хлор-3-(4-нітрофреніл)-аліліден]-4-оксо-2тіоксотіазолідин-3-іл\}-пропанову кислоту (1) та -сукцинатну кислоту (3) як сполуки-хіти з виразною активністю щодо вірусу Варіцелла-Зостер (значення індексу селективності 27 та 38 відповідно).
\end{abstract}

\section{СКРИНИНГ ПРОТИВОВИРУСНОЙ АКТИВНОСТИ В РЯДУ С5 И N3 ЗАМЕЩЕННЫХ ПРОИЗВОДНЫХ 4-ТИАЗОЛИДИНОНОВ \\ Д.В.Каминский}

Ключевые слова: 4-тиазолидиноны; скринине; противовирусная активность

Показана перспективность поиска противовирусных агентов среди производных 4-тиазолидинонов, отмечены оптимальные направления оптимизации структуры базового гетероцикла - положения C5 и N3. B результате проведенного скрининга (в рамках международной программы Antimicrobial Acquisition \& Coordinating Facility) установлены значения противовирусной активности исследуемых 5-замещенных-4-тиазолидинонов, которые содержат карбоксильную группу (или ее производные) в заместителе положения N3 относительно широкого спектра вирусов. Выделено ряд активных соединений, которые могут рассматриваться как перспективные структуры при дизайне агентов, воздействующих на вирусы гриппа, а также идентифицировано 3-\{5-[2-хлор-3-(4-нитрофенил)-алилиден]-4-оксо2-тиоксотиазолидин-3-ил\}-пропановую кислоту (1) и -сукцинатную кислоту (3) как соединения-хиты с выразительной активностью относительно вируса Варицелла-Зостер (значения индекса селективности 27 и 38 соответственно).

The search for new biologically active compounds based on the 4-thiazolidinone core [1-3] within different approaches and strategies [4] is successfully implemented over last decades. This is reflected in a large number of patents for biologically active compounds and introduction of 4-thiazolidinone-based drugs (such as glitazones - PPARs $\gamma$-agonist; Epalrestat, etc.) to the market. Thus, 4-thiazolidinones are considered as examples of privileged heterocycles in modern medical/pharmaceutical chemistry [5]. The "classical" directions in the area of development of drug-like small molecules among 4-thiazolidinones are the search of new antimicrobial, anticancer, antidiabetic, anti-inflammatory and antiviral agents.
Following the diversity of 4-thiazolidinones; the possibility of bioisosteric replacement, and chemical modification of the basic scaffolds a large number of 4-thiazolidinone sub-types (e.g., derivatives of 2,4-thiazolidinedione, rhodanine, 2 -amino(imino)4-thiazolidinone, 5-ylidene-4-thiazolidinones, etc.) are described. Among the subtypes mentioned 5-ylidene4-thiazolidinones and 4-thiazolidinone-3-carboxylic acids are the most studied and promising 4-thiazolidinones in the context of creating new drug-like molecules $[2,3,6]$. These sub-types represent the main important directions of the chemical modification of the 4-thiazolidinone core, namely positions $\mathrm{C} 5$ and N3. It have been found that the presence of the ylidene 
moiety in position $\mathrm{C} 5$ is desirable and often crucial for the biological effect of 4-thiazolidinones and its value $[2,7]$. On the other hand, introduction of substituents (mainly those containing a carboxyl group) in position $\mathrm{N} 3$ is regarded as the chemical route to the design of new compounds with a significant biological activity, but also considered as one of the approaches to decreasing toxicity of new compounds [8]. It should be also noted that 5-ylidene-4-thiazolidinone-3-carboxylic acids are among the preferred hitcompounds when using various in silico approaches with the subsequent experimental confirmation of the high affinity level to numerous biological targets [9]. Such findings are often criticized because of reffering 4-thiazolidiones to the so-called "frequent hitters" or "pan assay interference compounds (PAINS)" ("frequent hits" are compounds assigned as high-affinity ligands to the set of biotargets and tend to have a low specificity) $[5,10,11]$. Although, the debate of the importance of such approach remains open.

The search for the antiviral agents among 4-thiazolidinone derivatives are mainly presented in two directions: the search for new anti-HIV agents and compounds for treating viral hepatitis. The target compounds, namely compounds bearing the C5-ylidene fragment and the carboxyl group containing a substituent in position $\mathrm{N} 3$, are low molecular weight inhibitors of a number of biological targets - HCV NS3 protease (hepatitis C virus protease NS3) and HCV NS3-4A protease (regarded as an analogue of HIV protease) [12]; NS5B polymerase [13, 14] and NS3(5) helicase; HIV RT (reverse transcriptase of HIV) [15]; RNA helicase DDX3 [16] HIV-1 integrase [17], etc. However, the screening study of the antiviral activity [18-20] is still an important phase in the search for new antiviral agents. Thus, the aim of the present study was the screening of the antiviral activity among the range of 4-thiazolidinones with substituents in positions $\mathrm{C} 5$ and N3.

\section{Results and Discussion}

For the screening study 4-thiazolidinones containing substituents in positions $\mathrm{C} 5$ and N3, namely 5-ylidene-4-thiazolidinone-3-carboxylic acids and their derivatives $(\mathbf{1 - 4}, \mathbf{9 - 2 9}, \mathbf{3 2})$ were selected from the inhome library of the Department of Pharmaceutical, Organic and Bioorganic Chemistry at Danylo Halytsky Lviv National Medical University. Some isosteric compounds belonging to 2,4-imidazolidinediones (hydantoins) (5-8) and 5 alkyl-4-thiazolidinones $(30,31)$ were involved into the study to estimate the structure-activity relationship. The target compounds were obtained by the methods described earlier: $i$ ) rhodanine derivatives $(1-3,10,17-28)$ - based on rhodanine-3-carboxylic acid with the subsequent modification of position C5 (under Knoevenagel condensation conditions) and carboxyl groups (under the re- action of acylation) [7, 21, 22]; ii) compounds 4-9, 11-16 - based on 2,4-thia(imida)zolidinedione with the subsequent modification of position C5 and N3 (under the reaction of alkylation or cyanoethylation) [7]; iii) compounds 29,30 - based on 2,4-thiazolidinedione-5-alkyl (idene)carboxylic acids with the subsequent modification of the carboxyl group (under the acylation reaction) and position N3 [7, 23] (Fig.).

The antiviral activity screening of the compounds synthesized was performed at the National Institute of Allergic and Infectious Diseases of the National Institute of Health (Bethesda, MD, USA) within the framework of the international programme - Antimicrobial Acquisition and Coordinating Facility (http:// www.niaid-aacf.org/). The study involved the standard assay protocols regarding coronavirus SARS [24], herpes viruses (Herpes simplex virus 1 and 2 (HSV-1, HSV-2)), varicella zoster virus (VZV), Epstein-Barr virus (EBV), cytomegalovirus (HCMV) [25], hepatitis C (HCV) [26], and B (HBV) viruses [27], as well as vaccinia virus (Vaccinia, Cowpox) and influenza viruses of type A and type B, adeno- and rhinovirus; viruses of biological weapons [28] - Dengue fever virus, yellow fever virus, Takaribe virus, Rift Valley Fever vitus, Venezuelan equine encephalitis virus (VEE), measles virus, pig influenza virus (PIV), respiratory syncytial virus (RSV). The results obtained (Tab. 1-4) basically show a low to moderate level of the antiviral activity of the compounds studied.

It should be noted that 3-\{5-[2-chloro-3-(4-nitrophenyl)-allylidene]-4-oxo-2-thioxothiazolidine-3-yl\}propanoic acid (1) and 3-\{5-[2-chloro-3-(4-nitrophenyl)-allylidene]-4-oxo-2-thioxothiazolidine-3-yl\}-succinic acid (3) exhibit marked activity levels against Varitsella-Zoster virus (chickenpox) with the selectivity index values SI (the ratio of the cytotoxic concentration to the inhibitory concentration) of 27 and 38 , respectively (Table 1 ). In our opinion, the presence of the "ciminal" (2-chloro-3-(4-nitrophenyl)-propenal) moiety in position $\mathrm{C} 5$ of the 4 -azolidinone core is the most probable determinant. It can be usefull for design of new compounds with the antiviral activity. It is also noted that introduction of the additional carboxyl group (position N3 of compounds 1-3) leads to a drastic decrease of cytotoxicity.

A number of compounds, namely 2-7, 16, 17, 25, $\mathbf{2 9}, \mathbf{3 1}$, is characterized by a significant effect in relation to influenza viruses (both type A and type B) with SI values within 1.2-3.5. It allows to consider them as powerfull candidates for further optimization (Table 2). In this study it is noted that complications of the C5 substituent (transferring from simple benzylidene fragments to substituted derivatives or compounds with the phenylpropenylidene fragment) is not an efficient approach to enhance the antiviral activity.

Evaluation of the antiviral activity against hepatitis $\mathrm{C}$ and $\mathrm{B}$ viruses was conducted for two compounds - 
<smiles>O=C(O)CN1C(=O)/C(=C/C(Cl)=C/c2ccc([N+](=O)[O-])cc2)SC1=S</smiles>

$1\left(\mathrm{CH}_{2}\right)_{2}$

$2\left(\mathrm{CH}_{2}\right)_{5}$<smiles>[R]c1ccc(/C=C2\[X]C(=O)N(CCC#N)C2=O)cc1</smiles><smiles>[Y]C1=[X]C(=C([R])[R])C(=O)N1[Y]C(=O)Nc1ccccc1</smiles>
$\mathrm{R}^{2}$ $\begin{array}{llllll}\mathbf{X} & \mathbf{Y} & \mathbf{R}^{1} & \mathbf{R}^{2} & \mathbf{A} & \mathbf{R}\end{array}$

$6 \mathrm{~N} \quad \mathrm{O} \quad \mathrm{H} \quad 4-\mathrm{MeOC}_{6} \mathrm{H}_{4} \quad \mathrm{CH}_{2} \quad 3-\mathrm{CF}_{3}$

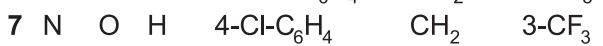

$8 \mathrm{~N} O \mathrm{H} \quad \mathrm{PhCHCH} \quad \mathrm{CH}_{2} \quad 4-\mathrm{Cl}$

$9 \mathrm{~S} \quad \mathrm{O} \quad \mathrm{H} \quad \mathrm{PhCHCH} \quad\left(\mathrm{CH}_{2}\right)_{2} \quad 3-\mathrm{CF}_{3}$

$10 \mathrm{~S} \quad \mathrm{~S} \quad \mathrm{H} \quad \mathrm{PhCHCH} \quad\left(\mathrm{CH}_{2}\right)_{2} \quad 4-\mathrm{SO}_{2} \mathrm{NH}_{2}$

$\begin{array}{llllll}11 \mathrm{~S} & \mathrm{O} & \mathrm{H} & 4-\mathrm{Et}_{-} \mathrm{C}_{6} \mathrm{H}_{4} & \mathrm{CH}_{2} & 4-\mathrm{F}\end{array}$

$12 \mathrm{~S} O \mathrm{H} \quad 3,4,5-(\mathrm{MeO})_{3} \mathrm{C}_{6} \mathrm{H}_{2} \mathrm{CH}_{2} \quad 4-\mathrm{SO}_{2} \mathrm{NH}_{2}$<smiles>[R]C=C1SC(=S)N(C2CC(=O)N([R])C2=O)C1=O</smiles>

$13 \mathrm{~S} O \mathrm{Me} \quad \mathrm{Me} \quad \mathrm{CH}_{2} \quad 4-\mathrm{F}$

$14 \mathrm{~S} O \mathbf{R}^{1+R^{2}}=\left(\mathrm{CH}_{2}\right)_{5} \quad \mathrm{CH}_{2} \quad 4-\mathrm{SO}_{2} \mathrm{NH}_{2}$

$15 \mathrm{~S} O \mathrm{R}^{1}+\mathbf{R}^{2}=\left(\mathrm{CH}_{2}\right)_{4} \quad \mathrm{CH}_{2} \quad 4-\mathrm{Cl}$

$16 \mathrm{~S} O \mathrm{R}^{1}+\mathrm{R}^{2}=$
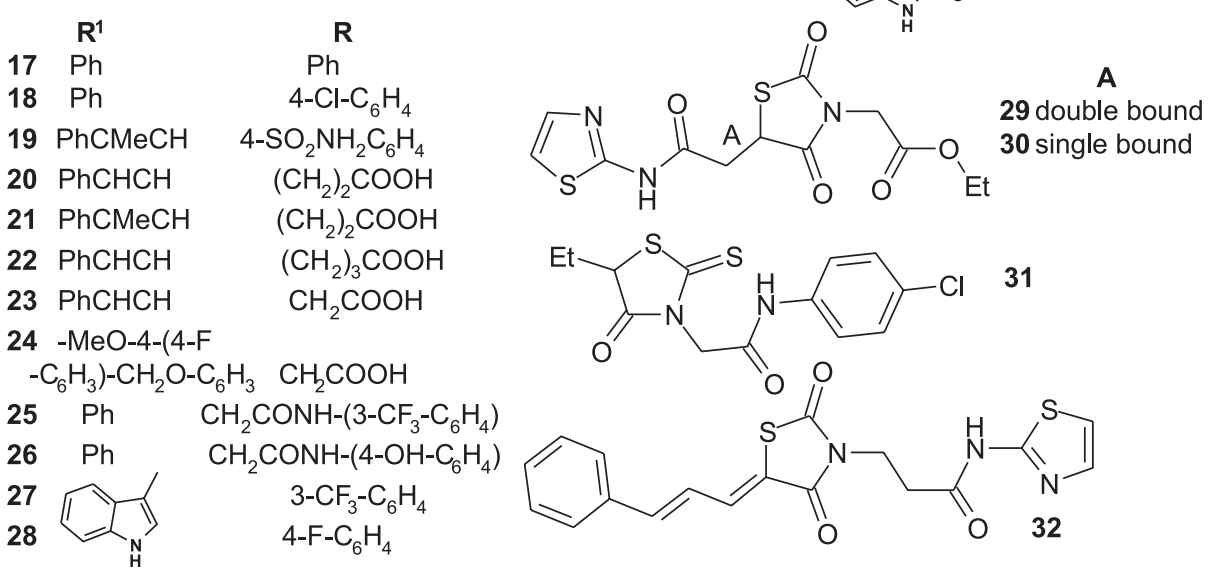<smiles>CCC1SC(=S)N(CC(=O)Nc2ccc(Cl)cc2)C1=O</smiles>

31

Fig. Structures of 4-thia(imida)zolidinones used in the study

Table 1

The antiviral activity of the compounds studied (screening data)

\begin{tabular}{|c|c|c|c|c|c|c|c|}
\hline Compound & Virus type, (assay) & Cell line & $\mathrm{EC}_{50}$ & $\mathrm{EC}_{90}$ & $\mathrm{CC}_{50}$ & $\mathrm{SI}$ & Cytotoxicity $\left(\mathrm{NR}, \mathrm{CC}_{50}\right)$ \\
\hline 1 & 2 & 3 & 4 & 5 & 6 & 7 & 8 \\
\hline \multirow{5}{*}{1} & HSV-1 (CPE) & HFF & $>12$ & $>12$ & 24 & $<2$ & \multirow{5}{*}{8} \\
\hline & HSV-2 (CPE) & HFF & $>12$ & $>12$ & 24 & $<2$ & \\
\hline & EBV (VCA Elisa) & Daudi & $>0.8$ & $>0.8$ & $>4$ & $<5$ & \\
\hline & HCMV (CPE) & HFF & $>2.4$ & $>2.4$ & 11 & $<4.6$ & \\
\hline & VZV (CPE) & HFF & 0.36 & 1.6 & 9.6 & 27 & \\
\hline \multirow{4}{*}{2} & HSV-1 (CPE) & HFF & $>0.5$ & $>0.5$ & 1.6 & $<3.2$ & \multirow{4}{*}{1,2} \\
\hline & HSV-2 (CPE) & HFF & $>0.5$ & $>0.5$ & 1.6 & $<3.2$ & \\
\hline & VZV (CPE) & HFF & $>2.4$ & $>2.4$ & 10.1 & $<4.2$ & \\
\hline & EBV (VCA Elisa) & Akata & $>0.8$ & $>0.8$ & 1 & $<1.3$ & \\
\hline \multirow{3}{*}{3} & HSV-1 (CPE) & HFF & $>300$ & $>300$ & $>300$ & 0 & \multirow{3}{*}{229} \\
\hline & HSV-2 (CPE) & HFF & $>300$ & $>300$ & $>300$ & 0 & \\
\hline & VZV (CPE) & HFF & 6.3 & 24.8 & 238 & 38 & \\
\hline \multirow{5}{*}{$\mathrm{ACV}$} & HSV-1 (CPE) & HFF & 1.1 & & & & \multirow{5}{*}{$>100$} \\
\hline & HSV-2 (CPE) & HFF & 1.0 & & & & \\
\hline & EBV (VCA Elisa) & Daudi & 0.2 & & & & \\
\hline & HCMV (CPE) & HFF & 0.2 & & & & \\
\hline & VZV (CPE) & HFF & 0.2 & & & & \\
\hline
\end{tabular}


Table continued

\begin{tabular}{|c|c|c|c|c|c|c|c|}
\hline 1 & 2 & 3 & 4 & 5 & 6 & 7 & 8 \\
\hline \multirow{2}{*}{4} & Cowpox (CPE) & HFF & - & - & - & - & \\
\cline { 2 - 7 } & Vaccinia (CPE) & HFF & $>300$ & $>300$ & $>300$ & $\mathbf{0}$ & \\
\hline \multirow{2}{*}{5} & Cowpox (CPE) & HFF & 245 & $>300$ & $>300$ & $>\mathbf{1 . 2}$ & \\
\cline { 2 - 7 } & Vaccinia (CPE) & HFF & 228 & $>300$ & $>300$ & $>\mathbf{1 . 3}$ & \\
\multirow{2}{*}{ DCV } & Cowpox (CPE) & HFF & \multirow{2}{*}{5.2} & 10.9 & $>317$ & & \multirow{2}{*}{317} \\
\cline { 2 - 7 } & Vaccinia (CPE) & HFF & & & \\
\hline
\end{tabular}

Hereinafter: ACV - acyclovir; CDV - cidogovir; CPE - inhibition of the cytopathic effect; NR - neutral red assay; $\mathrm{EC}_{50(90)}$ - concentration of the compound inhibiting virus replication by $50 \%(90 \%)$; $\mathrm{CC}_{50}$ - concentration of the compound reducing cells survival by $50 \%(\mu \mathrm{g} / \mathrm{mL})$.

24 and 30. These compounds do not possess a significant antiviral activity. The $\mathrm{EC}_{50}$ values were $>10$ and $\mathrm{CC}_{50}-98$ and $>100 \mu \mathrm{g} / \mathrm{mL}$, respectively, (compounds 24 and 30 ) in the hepatitis B virus assay (visual assay, control - 3TC). The percentage inhibition of HCV (replicon RNA) / Huh7 ET was determined for the anti-HCV activity evaluation of the compounds in the concentration of $20 \mathrm{mcM}$ in relation to hepatitis C. It was 0 and $83 \%$ for compounds $\mathbf{2 4}$ and $\mathbf{3 0 \text { , }}$ respectively. The studies of cytotoxicity allowed to determine the selectivity index of action. However, SI values were less than 1 ; and it was not a sufficient argument for further study of these compounds.

A moderate antiviral effect of compounds 14, 16, 21 and $\mathbf{3 1}$ has been found as a result of screening of new agents against the severe acute respiratory syndrome better known as SARS. Among the compounds mentioned 2-[5-(isatinylidene)-2,4-thiazoli-

Table 2

The antiviral activity of the compounds studied (flu-panel)

\begin{tabular}{|c|c|c|c|c|c|c|c|c|c|c|c|c|}
\hline \multirow{3}{*}{ Compound } & \multicolumn{3}{|c|}{$\begin{array}{c}\mathrm{A} H_{1} N_{1} \\
\text { Solomon Islands/03/2006 }\end{array}$} & \multicolumn{3}{|c|}{$\begin{array}{c}\mathrm{A} \mathrm{H}_{3} \mathrm{~N}_{2} \\
\text { Wisconsin /67/2005 }\end{array}$} & \multicolumn{3}{|c|}{$\begin{array}{c}\mathrm{A} H_{5} N_{1} \\
\text { Vietnam/1203/2004H }\end{array}$} & \multicolumn{3}{|c|}{$\begin{array}{c}\text { B } \\
\text { Malaysia/2506/2004 }\end{array}$} \\
\hline & \multicolumn{12}{|c|}{ (NR, MDCK) } \\
\hline & $\mathrm{EC}_{50}$ & $\mathrm{IC}_{50}$ & $\mathrm{SI}$ & $\mathrm{EC}_{50}$ & $I C_{50}$ & SI & $\mathrm{EC}_{50}$ & $I C_{50}$ & SI & $\mathrm{EC}_{50}$ & $I C_{50}$ & SI \\
\hline 2 & $>3$ & 3 & 0 & $>2.8$ & 2.8 & 0 & 0.32 & 0.86 & 2.7 & 0.34 & 1.1 & 3.3 \\
\hline 3 & $>100$ & $>100$ & 0 & $>100$ & $>100$ & 0 & 43 & $>100$ & $>2.3$ & 29 & $>100$ & $>3.5$ \\
\hline 4 & $>100$ & $>100$ & 0 & 80 & $>100$ & $>1.3$ & 34 & $>100$ & $>3$ & $>100$ & $>100$ & 0 \\
\hline 5 & 31 & $>100$ & $>3.2$ & 91 & $>100$ & $>1.1$ & 35 & $>100$ & $>2.8$ & 31 & $>100$ & $>3.2$ \\
\hline 6 & - & - & - & - & - & - & 38 & $>100$ & $>2.6$ & 40 & $>100$ & $>2.5$ \\
\hline 7 & - & - & - & - & - & - & $>29$ & 29 & 0 & 32 & 48 & 1.5 \\
\hline 8 & - & - & - & - & - & - & $>27$ & 27 & 0 & $>25$ & 25 & 0 \\
\hline 12 & - & - & - & - & - & - & $>100$ & $>100$ & 0 & $>100$ & $>100$ & 0 \\
\hline 14 & $>82$ & 82 & 0 & 32 & $>100$ & $>3.2$ & $>100$ & $>100$ & 0 & $>93$ & 93 & 0 \\
\hline 16 & - & - & - & 32 & 59 & 1.9 & 30 & $>100$ & $>3.3$ & 29 & $>100$ & $>3.4$ \\
\hline 17 & - & - & - & - & - & - & $>100$ & $>100$ & 0 & 76 & $>100$ & $>1.3$ \\
\hline 19 & - & - & - & - & - & - & $>30$ & 30 & 0 & $>33$ & 33 & 0 \\
\hline 20 & $>32$ & 32 & 0 & $>26$ & 26 & 0 & - & - & - & $>32$ & 32 & 0 \\
\hline 21 & - & - & - & - & - & - & $>27$ & 27 & 0 & 31 & 32 & 1 \\
\hline 24 & - & - & - & - & - & - & $>29$ & 29 & 0 & 32 & 33 & 1 \\
\hline 25 & - & - & - & - & - & - & 45 & $>100$ & $>2.2$ & 39 & 61 & 1.6 \\
\hline 27 & - & - & - & - & - & - & $>9$ & 9 & 0 & $>11$ & 11 & 0 \\
\hline 28 & - & - & - & - & - & - & $>36$ & 36 & 0 & $>32$ & 32 & 0 \\
\hline 29 & 53 & $>100$ & $>1.9$ & $>100$ & $>100$ & 0 & 37 & $>100$ & $>2.7$ & 58 & $>100$ & $>1.7$ \\
\hline 30 & $>100$ & $>100$ & 0 & $>100$ & $>100$ & 0 & $>100$ & $>100$ & $>100$ & $>100$ & 0 & \\
\hline 31 & $>100$ & $>100$ & 0 & 36 & $>100$ & $>2.8$ & $>100$ & $>100$ & 0 & $>100$ & $>100$ & 0 \\
\hline
\end{tabular}

In parentheses - assay, cell line. 
The antiviral activity of the compounds studied (different virus types)

\begin{tabular}{|c|c|c|c|c|c|c|c|c|c|c|c|c|}
\hline \multirow{2}{*}{$\begin{array}{l}\overline{0} \\
\bar{c} \\
0 \\
\stackrel{0}{\varepsilon} \\
\overline{0}\end{array}$} & \multicolumn{3}{|c|}{$\begin{array}{c}\text { Adenovirus } \\
65089 / \text { Chicago (NR, A-549) }\end{array}$} & \multicolumn{3}{|c|}{$\begin{array}{l}\text { Rhinovirus HGP } 2 \\
\text { (NR, HeLa Ohio-1) }\end{array}$} & \multicolumn{3}{|c|}{$\begin{array}{l}\text { PIV 14702 } \\
\text { (NR, MA-104) }\end{array}$} & \multicolumn{3}{|c|}{$\begin{array}{c}\text { RSV-A A2 } \\
\text { (NR, MA-104) }\end{array}$} \\
\hline & $\mathrm{EC}_{50}$ & $\mathrm{IC}_{50}$ & SI & $\mathrm{EC}_{50}$ & $\mathrm{IC}_{50}$ & SI & $\mathrm{EC}_{50}$ & $\mathrm{IC}_{50}$ & SI & $\mathrm{EC}_{50}$ & $\mathrm{IC}_{50}$ & SI \\
\hline 14 & $>39$ & 39 & 0 & 32 & 34 & 1.1 & $>49$ & 49 & 0 & $>48$ & 48 & 0 \\
\hline 16 & - & - & - & - & - & - & $>46$ & 46 & 0 & $>43$ & 43 & 0 \\
\hline 20 & - & - & - & $>100$ & 3 & 0 & - & - & - & - & - & - \\
\hline 29 & $>100$ & $>100$ & 0 & $>100$ & $>100$ & 0 & - & - & - & - & - & - \\
\hline \multirow[t]{3}{*}{31} & $>40$ & 40 & 0 & $>100$ & $>100$ & 0 & $>100$ & $>100$ & 0 & $>100$ & $>100$ & 0 \\
\hline & \multicolumn{3}{|c|}{$\begin{array}{l}\text { Measles virus Chicago } \\
(\mathrm{NR}, \mathrm{CV}-1)\end{array}$} & \multicolumn{3}{|c|}{$\begin{array}{l}\text { Yellow fever virus } \\
\text { MP-12 (NR, Vero 76) }\end{array}$} & \multicolumn{3}{|c|}{$\begin{array}{c}\text { Tacaribe } \\
\text { TRVL } 11573 \text { (NR, Vero 76) }\end{array}$} & \multicolumn{3}{|c|}{$\begin{array}{l}\text { Rift Valley Fever } \\
\text { MP-12 (NR, Vero 76) }\end{array}$} \\
\hline & $\mathrm{EC}_{50}$ & $\mathrm{IC}_{50}$ & $\mathrm{SI}$ & $\mathrm{EC}_{50}$ & $\mathrm{IC}_{50}$ & $\mathrm{SI}$ & $\mathrm{EC}_{50}$ & $I C_{50}$ & $\mathrm{SI}$ & $\mathrm{EC}_{50}$ & $\mathrm{IC}_{50}$ & $\mathrm{SI}$ \\
\hline 4 & - & - & - & $>26$ & 26 & 0 & $>100$ & $>100$ & 0 & $>100$ & 100 & 0 \\
\hline 5 & - & - & - & 25 & 26 & 1 & $>26$ & 26 & 0 & $>71$ & 71 & 0 \\
\hline 13 & - & - & - & $>58$ & 58 & 0 & $>100$ & $>100$ & 0 & - & - & - \\
\hline 14 & $>63$ & 63 & 0 & - & - & - & - & - & - & - & - & - \\
\hline 15 & - & - & - & - & - & - & 17 & 77 & 4.4 & $>30$ & 30 & 0 \\
\hline 16 & $>46$ & 46 & 0 & - & - & - & - & - & - & - & - & - \\
\hline 20 & $>10$ & 4.8 & 0 & - & - & - & - & - & - & - & - & - \\
\hline \multirow[t]{3}{*}{29} & - & - & - & $>79$ & 79 & 0 & $>51$ & 51 & 0 & - & - & - \\
\hline & \multicolumn{3}{|c|}{$\begin{array}{c}\text { Denge virus } \\
\text { New Guinea (NR, Vero) }\end{array}$} & \multicolumn{3}{|c|}{$\begin{array}{l}\text { VEE TC-83, } \\
\text { (NR, Vero) }\end{array}$} & & & & & & \\
\hline & $\mathrm{EC}_{50}$ & $\mathrm{IC}_{50}$ & $\mathrm{SI}$ & $\mathrm{EC}_{50}$ & $\mathrm{IC}_{50}$ & $\mathrm{SI}$ & & & & & & \\
\hline 4 & 84 & $>100$ & $>1.2$ & $>100$ & $>100$ & 0 & & & & & & \\
\hline 5 & $>100$ & $>100$ & 0 & $>100$ & $>100$ & 0 & & & & & & \\
\hline
\end{tabular}

Table 4

The results of anti-SARS screening (Urbani/Vero76, Neutral Red assay)

\begin{tabular}{|c|c|c|c|c|c|c|c|c|c|c|c|}
\hline Compound & $\mathrm{EC}_{50}$ & $\mathrm{IC}_{50}$ & $\mathrm{SI}$ & Compound & $\mathrm{EC}_{50}$ & $\mathrm{IC}_{50}$ & $\mathrm{SI}$ & Compound & $\mathrm{EC}_{50}$ & $\mathrm{IC}_{50}$ & $\mathrm{SI}$ \\
\hline 1 & $<10$ & $<10$ & 0 & 12 & $>100$ & $>100$ & 0 & 23 & $>100$ & 94 & 0 \\
\hline 2 & $>0.34$ & 0.34 & 0 & 14 & 30 & 38 & 1,3 & 24 & $<10$ & $<10$ & 0 \\
\hline 3 & $>33$ & 33 & 0 & 16 & 27 & $>100$ & $>3.7$ & 25 & 80 & 15 & 0 \\
\hline 6 & $>100$ & $>100$ & 0 & 17 & 58 & 90 & 2 & 26 & $>100$ & $>100$ & 0 \\
\hline 7 & $>100$ & $>100$ & 0 & 18 & 46 & 100 & 2 & 27 & 79 & 100 & 1 \\
\hline 8 & $>100$ & $>100$ & 0 & 19 & $>100$ & $>100$ & 0 & 29 & 87 & $>100$ & $>1.1$ \\
\hline 9 & 84 & $>100$ & $>1$ & 20 & 69 & 49 & 0 & 30 & $>100$ & $>100$ & 0 \\
\hline 10 & 20 & $<10$ & 0 & 21 & $<10$ & 19 & $>2$ & 31 & 56 & $>100$ & $>1.8$ \\
\hline 11 & $>100$ & $>100$ & 0 & 22 & 83 & 49 & 0 & 32 & $>100$ & 86 & 0 \\
\hline
\end{tabular}

dinedione-3-yl]-N-(4-fluorophenyl)-acetamide (16) is characterized by the highest selectivity index, $\mathrm{SI}>3.7$ (Table 4).

\section{Conclusions}

The screening of a number of 4-thiazolidinones with substituents in positions $\mathrm{C} 5$ and $\mathrm{N} 3$ (as the most promising directions of the 4-thiazolidinone structure optimisation) against a wide range of viruses has been carried out. It has been found that the compounds under research possess relatively low levels of the antiviral activity. However, some active compounds have been identified. The compound structures can be considered as promising basis for further modification in searching for antiviral agents. Among the compounds tested 3-\{5-[2-chloro-3-(4-nitrophenyl)-allylidene]4-oxo-2-thioxothiazolidinone-3-yl\}-propanoic (1) and 3-\{5-[2-chloro-3-(4-nirtophenyl)-allylidene]-4-oxo- 
2-thioxothiazolidinone-3-yl\}-succinic acids (3) show (VZV) with the selectivity index values - 27 and 38, a significant activity against Varitsella-Zoster virus

\section{respectively.}

\section{References}

1. Tripathi A. C., Gupta S. J., Fatima, G. N., Sonar P. K., Verma A., Saraf S. K. European Journal of Medicinal Chemistry, 2014, Vol. 72, pp.52-77. doi 10.1016/j.ejmech.2013.11.017.

2. Lesyk R. B., Zimenkovsky B. S. Current Organic Chemistry, 2004, Vol. 8, No.16, pp.1547-1577. doi:10.2174/1385272043369773.

3. Lesyk R. B., Zimenkovsky B. S., Kaminskyy D. V., Kryshchyshyn A. P., Havryluk D. Ya., Atamanyuk D. V., Subtel'na I. Yu., Khyluk D. V. Biopolymer and Cell, 2011, Vol. 27, No.2, pp.107-117. doi: 10.7124/bc.000089.

4. Zhang Y., Wang S., Wu S., Zhu S., Dong G., Miao Z., Yao J., Zhang W., Sheng C., Wang W. ACS Combinatorial Science, 2013, Vol. 15, No.6, pp.298-308. doi: $10.1021 /$ co400022r.

5. Tomašic T., Mašic L. Expert Opinion on Drug Discovery, 2012, Vol. 7. No.7, pp.549-560. doi: 10.1517/17460441.2012.688743.

6. Kaminskyy D. V., Lesyk R. B. Biopolymer and Cell, 2010, Vol. 26, No.2, pp.136-145. doi: 10.7124/bc.000150.

7. Kaminskyy D., Zimenkovsky B., Lesyk R. European Journal of Medicinal Chemistry, 2009, Vol. 44, No.9. pp.3627-3636. doi: 10.1016/j.ejmech.2009.02.023.

8. Bhat B. A., Ponnala S., Sahu D. P. Tiwari P., Tripathi B. K., Srivastava A. K. Bioorganic and Medicinal Chemistry, 2004, Vol. 12, No.22, pp.5857-5864. doi: 10.1016/j.bmc.2004.08.031.

9. Volynets G. P., Bdzhola V. G., Golub A. G., Synyugin A. R., Chekanov M. A., Kukharenko O. P., Yarmoluk S. M. European Journal of Medicinal Chemistry, 2013, Vol.61, pp.104-115. doi: 10.1016/j.ejmech.2012.09.022.

10. Baell J. B., Holloway G. A. Journal of Medicinal Chemistry, 2010, Vol. 53, No.7, pp.2719-2740. doi: 10.1021/jm901137j.

11. Mendgen T., Steuer C., Klein C. D. Journal of Medicinal Chemistry, 2012, Vol. 55, No.2, pp.743-753. doi 10.1021/jm201243p.

12. Sing W. T., Lee C. L., Yeo S. L., Lim S. P., Sim M. Biorganic and Medicinal Chemistry Letter, 2001, Vol. 11, No.2, pp.91-94. doi: 10.1016/S0960-894X(00)00610-7.

13. Vermehren J., Sarrazin C. Clinical Microbiology and Infection, 2011, Vol. 17, No.2, pp.122-134. doi: 10.1111/j.1469-0691.2010.03430.x.

14. Kucukguzel I., Satılmis G., Gurukumar K. R., Basu A., Tatar E., Nichols D. B., Talele T. T., Kaushik-Basu N. European Journal of Medicinal Chemistry, 2013, Vol. 69, pp.931-941. doi: 10.1016/j.ejmech.2013.08.043

15. Barreca M. L., Chimirri A., De Clercq E., De Luca L., Monforte A. M., Monforte P., Rao A., Zappala M. Il Farmaco, 2003, Vol. 58, No.3, pp.259-263. doi: 10.1016/S0014-827X(03)00024-7.

16. Maga G., Falchi F., Garbelli A., Belfiore A., Witvrouw M., Manetti F., Botta M. Journal of Medicinal Chemistry, 2008, Vol. 51, No.21, pp.6635-6638. doi: 10.1021/jm8008844.

17. Dayam R., Gundla R., Al-Mawsawi L. Q., Neamati N. Medicinal Research Reviews, 2008, Vol. 28, No.1, pp.118-154. doi: 10.1002/med.20116.

18. Havrylyuk D., Zimenkovsky B., Vasylenko O., Lesyk R. Journal of Heterocyclic Chemistry, 2013, Vol. 50, No.S1, pp.E55-E62. doi: 10.1002/jhet.1056.

19. Havrylyuk D., Khyrkov S., Atamanyuk V., Zimenkovsky B., Lesyk R. Aktualni Pytannia Farmacevtychnoi Nauky i Praktyky - Current Issues in Pharmacy and Medicine: Science and Practice, 2012, Vol. 10, No.3, pp.32-41. (in Ukrainian). http://pharmed.zsmu.edu.ua/issue/viewIssue/1480/236.

20. Gavrylyuk D. Ya., Zimenkovsky B. S., Vasylenko O. M., Lesyk R. B. Zhurnal Organichnoi i Farmacevtychnoi Chimii - Journal of Organic and Pharmaceutical Chemistry, 2009, Vol. 7, No.1(25), pp.42-47. (in Ukrainian). http://ru.nuph.edu.ua/files/2012/03/\%D0\%96\%D0\%9E\%D0\%A4\%D0\%A5-1-2009-42-47.pdf.

21. Kaminskyy D. V., Roman O. M., Atamanyuk D. V., Lesyk R. B. Zhurnal Organichnoi i Farmacevtychnoi Chimii - Journal of Organic and Pharmaceutical Chemistry, 2006, Vol. 4, No.1(13), pp.41-48. (in Ukrainian). http://dspace.nbuv.gov.ua/bitstream/handle/123456789/42099/05-Kaminsky. pdf? sequence $=1$.

22. Kaminskyy D. V., Lesyk R. B. Farmacevtychyy Zhurnal - Pharmaceutical Journal, 2008, No.3, pp.70-78. (in Ukarinian).

23. Zimenkovskii B. S., Kutsyk R. V., Lesyk R. B., Matyichuk V. S., Obushak N. D., Klyufinska T. I. Pharmaceutical Chemistry Journal, 2006, Vol. 40, No.6, pp.303-306. doi: 10.1007/s11094-006-0115-6.

24. Severson W. E., Shindo N., Sosa M., Fletcher T., White E. L., Ananthan S., Jonsson C. B. Journal of Biomolecular Screening, 2007, Vol. 12, No.1, pp.33-40. doi: 10.1177/1087057106296688.

25. Cotarelo M., Catalán P., Sánchez-Carrillo C., Menasalvas A., Cercenado E., Tenorio A., Bouza E. Journal of Antimicrobial Chemotherapy, 1999, Vol. 44, No.5, pp.705-708. doi: 10.1093/jac/44.5.705.

26. Krieger N., Lohmann V., Bartenschlager R. Journal of Virology, 2001, Vol. 75, No.10, pp.4614-4624. doi: 10.1128/JVI.75.10.4614-4624.2001.

27. Korba B. E., Gerin J. L. Antiviral Research, 1992, Vol. 19, No.1, pp.55-70. doi: 10.1016/0166-3542(92)90056-B.

28. Sidwell R. W., Huffman, J. H. Applied Microbiology, 1971, Vol. 22, No.5, pp. 797-801. http://aem.asm.org/content/22/5/797.full.pdf+html

Надійшла до редакції 27.10.2014 р.

\section{Acknowledgements}

We are grateful to Dr. Robert Reynolds and his colleagues from Virology Branch, Division of Microbiology of the National Institute of Allergy and Infectious Diseases, NIH, USA for in vitro evaluation of the antiviral activity within AACF programme. 